\title{
Multilevel Methods for Eigenspace Computations in Structural Dynamics
}

\author{
Ulrich Hetmaniuk $^{1}$ and Richard B. Lehoucq ${ }^{1}$ \\ 1 Sandia National Laboratories, ulhetma@sandia.gov. Sandia is a multiprogram \\ laboratory operated by Sandia Corporation, a Lockheed Martin Company, for \\ the U.S. Department of Energy under contract DE-AC04-94AL85000. \\ 2 Sandia National Laboratories, rblehou@sandia.gov. Sandia is a multiprogram \\ laboratory operated by Sandia Corporation, a Lockheed Martin Company, for \\ the U.S. Department of Energy under contract DE-AC04-94AL85000.
}

Summary. Dynamic analysis of three-dimensional structures frequently involves finite element discretizations with over a million unknowns. The discretization is typically used to enable engineering analysis so that a reduced order model (ROM) is advantageous or even necessary. The standard reduction approach is accomplished with a modal truncation, which requires a costly partial eigensolution but reduces the number of unknowns by orders of magnitude. The cost of the partial eigensolution required for modal truncation increases as the frequency range for the analysis increases because the number of eigenpairs needed can easily reach into the thousands.

An approach for computing the partial eigensolution is to use a shift-invert Lanczos method or a preconditioned eigensolver. Both methods avail themselves of a multilevel preconditioner. Alternatives in computing the eigenspace needed for the ROM include component mode synthesis (CMS) techniques that subdivide the structure into numerous substructures. Modes associated with these substructures are used to approximate the eigenvectors. Our paper reviews recent work in multilevel methods for eigenspace computations in structural dynamics where both the standard approach and CMS based techniques are employed.

\section{Introduction}

The goal of our paper is to provide a brief review of our work in multilevel methods for eigenspace computations in structural dynamics. Our review is not meant to be exhaustive and so we apologize for relevant work that we do not discuss. In particular, our interest is in multilevel algorithms for the numerical solution of the resulting algebraic generalized eigenvalue problem arising from the finite element discretization of three dimensional structures. Our interest is also restricted in methods that are scalable, both with respect to the mesh size and the number of processors of extremely large distributed memory architecture. We start our paper by a formal discussion of the origin of the eigenvalue problem. 
The dynamic analysis of a three-dimensional structure is typically modeled by the hyperbolic PDE

$$
\rho u_{t t}-\mathcal{E}(u)=f(t) \text { in } \Omega
$$

where $\mathcal{E}(u)$ is an elliptic differential equation, $\rho$ is the mass density, $f$ is some load function of interest, and both $u$ and $f$ are vector functions. For example, if $\mathcal{E}(u)=\operatorname{div} \sigma(u)$ then $\sigma(u)$ is the linearized stress tensor associated with the displacement $u$ obtained from the inner product between the fourth-order tensor of elastic coefficients $\mathcal{C}_{i j k l}$ and the linearized strain tensor

$$
\epsilon_{k l}(u)=\frac{1}{2}\left(\frac{\partial u_{k}}{\partial x_{l}}+\frac{\partial u_{l}}{\partial x_{k}}\right) .
$$

Because our interest is in a formal presentation of the origin of the eigenvalue problem in structural dynamics, we assume that appropriate boundary conditions and initial conditions are specified on some simply connected domain three-dimensional domain $\Omega$.

If we take the Fourier transform of (1), then the time derivative is eliminated and the Helmholtz differential equation

$$
-\omega^{2} \rho u_{\omega}-\mathcal{E}\left(u_{\omega}\right)=f_{\omega}
$$

results. The standard solution approach is to project this Helmholtz equation onto the modal subspace given by the eigenvalues and eigenvectors of the vibrational problem

$$
-\mathcal{E}(u)=\lambda \rho u \text { in } \Omega
$$

with the same boundary conditions as (8) where $\lambda$ is the square of the natural frequency $\omega$ and the subscript $\omega$ is neglected for ease of notation.

A finite element discretization of the weak form of the vibrational problem (2) leads to the generalized eigenvalue problem

$$
\mathbf{K} \mathbf{u}^{h}=\mathbf{M} \mathbf{u}^{h} \lambda^{h}
$$

where $\mathbf{K}$ and $\mathbf{M}$ are the stiffness and mass matrices of order $n$ respectively that represent the elastic and inertial properties of a structure. Assuming appropriate boundary conditions, both matrices are symmetric and positive definite. (For an introduction to finite element methods in structural dynamics, the reader is referred to $[11]$.)

Three dimensional structures frequently involve finite element discretizations with over with well over one million unknowns and so a computationally intensive linear algebra problem ensues. Modal truncation or computing anything but a small subset of the eigenvalues and eigenvectors of (3) is prohibitive. Nevertheless, 100 1000 eigenpairs may be needed. The discrete modal subspace is used to generate a reduced order model (ROM) for (1) so that engineering analysis is possible. Typical analysis include an harmonic response or transient simulation (see [11] and the references therein for further details). Modal truncation is justified because eigenfunctions associated with larger frequencies have a much lower participation in the response than lower ones. But there is an additional justification when a finite element discretization is used. If enough modes are retained that the error associated with modal truncation is no larger than the discretization error, the cost of computing eigenvector approximations may be dramatically reduced without a significant loss of accuracy. 
The error in the eigenspace approximations computed is composed of an error due to discretization and the algorithm used to compute the discrete eigenspace. We first briefly discuss the former error. Standard results from finite element theory [2] give that

$$
\lim _{h \rightarrow 0} \lambda_{i}^{h}=\lambda_{i}, \quad \lim _{h \rightarrow 0} \lambda_{n}^{h}=+\infty
$$

where $0<\lambda_{1} \leq \lambda_{2} \leq \cdots \leq \lambda_{i}$. Note that $n \rightarrow+\infty$ as $h \rightarrow 0$. If $p$ is the degree of the finite elements, then Strang and Fix [37] give the following a-priori error estimates

$$
\lambda_{i} \leq \lambda_{i}^{h} \leq \lambda_{i}\left(1+C h^{2 p} \lambda_{i}^{p}\right) \quad \text { and } \quad\left\|u_{i}-u_{i}^{h}\right\|_{H^{1}(\Omega)} \leq C h^{p} \lambda_{i}^{p / 2} .
$$

These estimates imply that the eigenvalue discretization error is the square of that of the eigenfunction. Note how both errors also increase as the eigenvalue approximated increases. Ideally, the error in the algorithm used to compute the discrete eigenspace should be no smaller than that of the discretization error.

The remainder of our paper reviews two multilevel approaches that can be used to generate the needed eigenspace. The first, multilevel as a preconditioner, uses multilevel techniques within a shift-invert Lanczos scheme or a preconditioned eigensolver. The second approach, multilevel applied to (3), decomposes the structure, via multilevel techniques, and computes approximations to the needed eigenspace from the modes of the decomposed structure. We will refer to these as multilevel approaches I and II, respectively, and these approaches are the subject of the next two sections.

\section{Multilevel Approach I}

Suppose a multilevel preconditioner $\mathbf{N}$ is available for the stiffness matrix $\mathbf{K}$. The preconditioner can be used within two types of algorithms:

- within the shift-invert Lanczos [15, 20] algorithm;

- within a gradient- or Newton-based method.

Replacements for the shift-invert Lanczos algorithm include gradient and Newton schemes that attempt to minimize the Rayleigh-quotient. The gradient schemes include conjugate gradient schemes $[4,18,21,24,27,30]$ and the Newton based schemes include the Davidson [13] based methods such as the Jacobi-Davidson algorithm [36]

The two types of algorithms perform a Rayleigh-Ritz analysis using a representation of the space

$$
\mathcal{S}_{m+1}\left(\mathbf{x}^{(0)}\right) \equiv \operatorname{Span}\left\{\mathbf{x}^{(0)}, \cdots, \mathbf{x}^{(m)}, \mathbf{N}^{-1} \mathbf{r}^{(m)}\right\},
$$

where $\mathbf{r}^{(m)}=\mathbf{M} \mathbf{x}^{(m)} \theta^{(m)}-\mathbf{K} \mathbf{x}^{(m)}$ and

$$
\mathbf{x}^{(m)} \equiv \min _{\mathbf{x}(\neq \mathbf{0}) \in \mathcal{S}_{m}} \frac{\mathbf{x}^{T} \mathbf{K} \mathbf{x}}{\mathbf{x}^{T} \mathbf{M} \mathbf{x}} \quad \text { and } \quad \theta^{(m)} \equiv \frac{\left(\mathbf{x}^{(m)}\right)^{T} \mathbf{K} \mathbf{x}^{(m)}}{\left(\mathbf{x}^{(m)}\right)^{T} \mathbf{M} \mathbf{x}^{(m)}}
$$

We remark that when $\mathbf{N}=\mathbf{K}$, then $\mathcal{S}_{m+1}\left(\mathbf{x}^{(0)}\right)$ is equal to the Krylov subspace

$$
\mathcal{K}_{m+1}\left(\mathbf{K}^{-1} \mathbf{M}, \mathbf{x}^{(0)}\right) \equiv \operatorname{Span}\left\{\mathbf{x}^{(0)}, \mathbf{K}^{-1} \mathbf{M} \mathbf{x}^{(0)}, \cdots,\left(\mathbf{K}^{-1} \mathbf{M}\right)^{m} \mathbf{x}^{(0)}\right\}
$$


a result that is easily established using mathematical induction. The shift-invert Lanczos iteration with initial vector $\mathbf{x}^{(0)}$ computes a representation for the Krylov subspace.

When $\mathbf{N} \approx \mathbf{K}$, then an inner iteration is needed within the shift-invert Lanczos iteration to approximately solve the linear systems with the coefficient matrix $\mathbf{K}$. This inner iteration is preconditioned with $\mathbf{N}$. The replacements for the shiftinvert Lanczos iteration avoid the requirement for an inner iteration so that a single application of $\mathbf{N}$ per outer iteration can be used.

Good preconditioners are a prerequisite for any of the algorithms to perform satisfactorily. If a multilevel preconditioner is used to solve the partial differential equation

$$
-\mathcal{E}(u)=g \text { in } \Omega
$$

for some load term $g$, is available, then this preconditioner is used for $\mathbf{N}$. A multilevel preconditioner for $\mathbf{K}$ is desirable because the rate of convergence of the resulting preconditioned conjugate gradient iteration is independent of the mesh size. Although less studied, preconditioned iterations for the eigenvalue problem should also be independent of the mesh size. The reader is referred to $[23,25,1]$ and $[31,32]$ for a review of the many issues involved and convergence theory, respectively. These five papers also contain numerous citations to the engineering and numerical analysis literature.

Salinas is a massively parallel implementation of finite element analysis for structural dynamics developed at Sandia National Laboratories. This capability is required for high fidelity validated models used in modal, vibrations, static and shock analysis of weapons systems. Salinas was a 2002 Gordon Bell Prize winner. A critical component of Salinas are scalable iterative linear algebra and eigensolvers. The modal analysis is computed with a shift-invert Lanczos method using parallel ARPACK $[26,29]$ and the FETI iterative linear solver $[17,16]$. The use of FETI for linear systems generated by the Sandia ASCI Salinas code is elucidated in [5]. The ARPACK-FETI combination also introduces deflation used when FETI computes the needed Lagrange multipliers. Because the Lanczos iteration used by ARPACK makes repeated calls to FETI, the projected conjugate iteration used for computing the Lagrange multipliers retains a history of vectors computed during each FETI invocation. After the first FETI call by ARPACK, the right-hand side in the projected conjugate iteration is first orthogonalized against this history of vectors. The number of projected conjugate iterations is therefore reduced as the number of Lanczos iterations needed by ARPACK increases.

Besides the capability developed for Salinas, the authors are not aware of any multilevel based modal analysis capabilities for use within 3D structural dynamics. Moreover, there is little information available, either experimental or theoretical, comparing the merits of shift-invert Lanczos methods with preconditioned eigensolvers when a large number, say a few hundred eigenvalues and eigenvectors are to be computed when a scalable preconditioner is available. The paper [1] compares a number of algorithms on several large-scale eigenvalue problems arising in structural dynamics when an algebraic multigrid preconditioner is available. One implication of [1] is that the cost of all the algorithms (and implementations) is asymptotic with the cost of maintaining numerical orthogonality of the number of eigenvector approximations required. Are there alternatives? 


\section{Multilevel Approach II}

The previous section described schemes where knowledge of the preconditioner by the eigensystem iteration is only required through its application on a vector (or block of vectors). Given that our goal is to compute hundreds of eigenvectors, perhaps this separation of preconditioner from eigen-iteration needs to be reconsidered given that the cost becomes dominated by maintaining numerical orthogonality. In contrast, the multilevel approaches in this section eliminate or minimize the number of orthogonalizations of fine grid vectors. This is accomplished by decomposing the domain into substructures or grids via projection. As in the previous section, there are two types of algorithms:

- avoiding iteration among the substructures or a sequence of grids;

- iteration among the substructures or a sequence of grids.

Component mode synthesis (CMS) techniques [22, 12] that originated in the aerospace engineering community and multigrid/multilevel schemes [28, 9] are examples of the two types of algorithms, respectively. We first discuss CMS techniques followed by a brief remarks on iteration-based approaches.

CMS schemes decompose a structure into numerous components (or substructures), determine component modes and then synthesize these modes that approximate a representation of the needed eigenspace. The reader is referred to [35] for a review of CMS methods from a structural dynamics perspective. Classical CMS techniques are properly viewed as approximation schemes and differ from domain decomposition algorithms that use iteration to solve a PDE. Although some iteration can be used to compute the component and synthesis modes, the goal is to generate approximations that aptly describe the low frequency modal subspace. Eigenvectors approximations constructed from these subdomains are used rather than the traditional eigenvector approximations computed via the techniques described in the previous section. We remark that the synthesis step is tantamount to an interface problem.

To make the process concrete, suppose that the structure $\Omega$ is divided into two subdomains $\Omega_{i}$ with the common interface $\Gamma$. One-way dissection on the union of the graphs of the mass and stiffness matrices reorders the $\mathbf{M}$ and $\mathbf{K}$ into

$$
\left[\begin{array}{ccc}
\mathbf{M}_{\Omega_{1}} & \mathbf{0} & \mathbf{M}_{\Omega_{1}, \Gamma} \\
\mathbf{0} & \mathbf{M}_{\Omega_{2}} & \mathbf{M}_{\Omega_{2}, \Gamma} \\
\mathbf{M}_{\Omega_{1}, \Gamma}^{T} & \mathbf{M}_{\Omega_{2}, \Gamma}^{T} & \mathbf{M}_{\Gamma}
\end{array}\right] \text { and }\left[\begin{array}{ccc}
\mathbf{K}_{\Omega_{1}} & \mathbf{0} & \mathbf{K}_{\Omega_{1}, \Gamma} \\
\mathbf{0} & \mathbf{K}_{\Omega_{2}} & \mathbf{K}_{\Omega_{2}, \Gamma} \\
\mathbf{K}_{\Omega_{1}, \Gamma}^{T} & \mathbf{K}_{\Omega_{2}, \Gamma}^{T} & \mathbf{K}_{\Gamma}
\end{array}\right]
$$

The unknowns associated with rows and columns identified by $\Gamma$ constitute a separator for the graph. The finite element nodes associated with this separator identify element boundaries that form the interface $\Gamma$. Block Gaussian elimination on $\mathbf{K}$ results in $\mathbf{V}^{T} \mathbf{K V}=\operatorname{diag}\left[\mathbf{K}_{\Omega_{1}} \mathbf{K}_{\Omega_{2}} \tilde{\mathbf{K}}_{\Gamma}\right]$, where

$$
\mathbf{V}=\left[\begin{array}{ccc}
\mathbf{I}_{n_{\Omega_{1}}} & \mathbf{0} & -\mathbf{K}_{\Omega_{1}}^{-1} \mathbf{K}_{\Omega_{1}, \Gamma} \\
\mathbf{0} & \mathbf{I}_{n_{\Omega_{2}}} & -\mathbf{K}_{\Omega_{2}}^{-1} \mathbf{K}_{\Omega_{2}, \Gamma} \\
\mathbf{0} & \mathbf{0} & \mathbf{I}_{n_{\Gamma}}
\end{array}\right] .
$$

The matrix 


$$
\tilde{\mathbf{K}}_{\Gamma}=\mathbf{K}_{\Gamma}-\sum_{i=1}^{2} \mathbf{K}_{\Omega_{i}, \Gamma}^{T} \mathbf{K}_{\Omega_{i}}^{-1} \mathbf{K}_{\Omega_{i}, \Gamma}
$$

is the Schur complement of $\operatorname{diag}\left[\mathbf{K}_{\Omega_{1}} \mathbf{K}_{\Omega_{2}}\right]$ in $\mathbf{K}$ and is the discrete equivalent of the Steklov-Poincaré operator. If we perform a congruence transformation on (3) with $\mathbf{V}$, then we obtain

$$
\mathbf{V}^{T} \mathbf{K V} \hat{\mathbf{u}}^{h}=\operatorname{diag}\left[\mathbf{K}_{\Omega_{1}} \mathbf{K}_{\Omega_{2}} \tilde{\mathbf{K}}_{\Gamma}\right] \hat{\mathbf{u}}^{h}=\mathbf{V}^{T} \mathbf{M V} \hat{\mathbf{u}}^{h} \lambda^{h} h,
$$

where $\mathbf{u}^{h}=\mathbf{V} \hat{\mathbf{u}}^{h}$ and the upper triangular part of the symmetric matrix $\mathbf{V}^{T} \mathbf{M V}$ is

$$
\left[\begin{array}{ccc}
\mathbf{M}_{\Omega_{1}} & \mathbf{0} & \mathbf{M}_{\Omega_{1}, \Gamma}-\mathbf{M}_{\Omega_{1}} \mathbf{K}_{\Omega_{1}}^{-1} \mathbf{K}_{\Omega_{1}, \Gamma} \\
\mathbf{0} & \mathbf{M}_{\Omega_{2}} & \mathbf{M}_{\Omega_{2}, \Gamma}-\mathbf{M}_{\Omega_{2}} \mathbf{K}_{\Omega_{2}}^{-1} \mathbf{K}_{\Omega_{2}, \Gamma} \\
\star & \star & \tilde{\mathbf{M}}_{\Gamma}
\end{array}\right]
$$

The matrix $\tilde{\mathbf{M}}_{\Gamma}$ is

$$
\mathbf{M}_{\Gamma}-\sum_{i=1}^{2}\left(\mathbf{K}_{\Omega_{i}, \Gamma}^{T} \mathbf{K}_{\Omega_{i}}^{-1} \mathbf{M}_{\Omega_{i}, \Gamma}+\mathbf{M}_{\Omega_{i}, \Gamma}^{T} \mathbf{K}_{\Omega_{i}}^{-1} \mathbf{K}_{\Omega_{i}, \Gamma}-\mathbf{K}_{\Omega_{i}, \Gamma}^{T} \mathbf{K}_{\Omega_{i}}^{-1} \mathbf{M}_{\Omega_{i}} \mathbf{K}_{\Omega_{i}}^{-1} \mathbf{K}_{\Omega_{i}, \Gamma}\right)
$$

and is the discrete version of a mass complement operator. If the columns of $\mathbf{Z}_{\Omega_{i}}$ and $\mathbf{Z}_{\Gamma}$ are eigenvectors for the substructure matrix pencils $\left(\mathbf{K}_{\Omega_{i}}, \mathbf{M}_{\Omega_{i}}\right)$ and $\left(\tilde{\mathbf{K}}_{\Gamma}, \tilde{\mathbf{M}}_{\Gamma}\right)$, respectively, then a Rayleigh-Ritz analysis is undertaken with

$$
\left[\begin{array}{ccc}
\mathbf{Z}_{\Omega_{1}} & 0 & -\mathbf{K}_{\Omega_{1}}^{-1} \mathbf{K}_{\Omega_{1}, \Gamma} \mathbf{Z}_{\Gamma} \\
0 & \mathbf{Z}_{\Omega_{2}} & -\mathbf{K}_{\Omega_{2}}^{-1} \mathbf{K}_{\Omega_{2}, \Gamma} \mathbf{Z}_{\Gamma} \\
0 & 0 & \mathbf{Z}_{\Gamma}
\end{array}\right]
$$

to obtain eigenspace approximations to (3). Savings occur when the number of substructure eigenvectors are small relative to the size of the substructure matrix pencils so that the number of columns of (13) is significantly less than the order of (3). For example, because our interest is in the smallest eigenvalues (and associated eigenvectors), only the smallest eigenvalues of the subdomain and substructures are retained. This process that retains only a subset of the substructure eigenpairs is called modal truncation.

We remark that the interface eigenvalue pencil $\left(\tilde{\mathbf{K}}_{\Gamma}, \tilde{\mathbf{M}}_{\Gamma}\right)$ represents the discrete $H^{1}(\Omega)$ (e.g. $\mathbf{K}$ orthogonal) projection of (3) onto the interface $\Gamma$. Therefore, the Rayleigh-Ritz analysis renders a diagonal representation of the stiffness matrix because the subdomain eigenvalue problems are also discrete $H^{1}(\Omega)$ projections of (3) onto $\Omega_{i}$. The projected mass matrix is not, however, diagonal. The projected mass matrix does possess the same block form as the reordered mass matrix of (9). Finally, independent of the Rayleigh-Ritz eigenvalue problem that determines the eigenspace approximations to (3), the resulting eigenvector approximations are $\mathbf{K}$ orthogonal - and this occurred without any explicit orthogonalization of the fine grid vectors.

Our previous discussion is an example of a fixed-interface CMS method that derives its name from the fact that the eigenvalue problems associated with $\Omega_{i}$ impose a homogeneous essential boundary condition along the interface $\Gamma$. Fixed-interface CMS methods can be posed for the weak formulation of the eigenvalue problem 
(2). The variational formulation of classical CMS techniques is due to Bourquin $[6,7,8]$. Asymptotic results for second order elliptic differential eigenvalue problems and their finite element discretization were derived. In three dimensions, for the case of two subdomains $\Omega_{i}$ separated by an interface $\Gamma$,

$$
0 \leq \lambda_{k}-\lambda_{k}^{N} \leq C(k)\left[\sum_{i=1}^{2} \frac{\varepsilon_{i}\left(s, k, N_{i}\right)}{\left(C_{i}\left(N_{i}\right) N_{i}^{2 / 3}\right)^{1+s}}+\frac{\varepsilon_{\Gamma}\left(\alpha, k, N_{\Gamma}\right)}{C_{\Gamma} N_{\Gamma}^{2 \alpha}}\right],
$$

gives the error in the eigenvalue approximation due to modal truncation, where $N=\left(N_{1}, N_{2}, N_{\Gamma}\right)$ is the triple composed of the number eigenmodes retained in $\Omega_{i}$ and $\Gamma$. The functions $C_{i}\left(N_{i}\right)$ are positive and increasing and depends essentially on the volume of $\Omega_{i}$. The constant $C_{\Gamma}$ is expected to increase when the measure of $\Gamma$ decreases. The functions $\varepsilon_{i}$ and $\varepsilon_{\Gamma}$ satisfy

$$
\lim _{N_{i} \rightarrow \infty} \varepsilon_{i}\left(s, k, N_{i}\right)=0, \quad \lim _{N_{\Gamma} \rightarrow \infty} \varepsilon_{\Gamma}\left(\alpha, k, N_{\Gamma}\right)=0 .
$$

The coefficient $\alpha$ determines the regularity of a solution in $H^{1+\alpha}(\Omega)$, and $0<s<$ $1 / 2$ is an artifact of the method of proof.

Our interest in reproducing (14) is to indicate the cost of solving threedimensional problems with fixed-interface CMS methods. We note the following. The subdomain error components are each inversely proportional to the product of $N_{i}^{2 / 3}$ and the volume of $\Omega_{i}$. The interface error is inversely proportional to the product of the measure (not volume) of $\Gamma$ and $N_{\Gamma}^{2 \alpha}$. Hence as the regularity of the interface modes increases, $\alpha$ increases, then the number of interface modes needed for a prescribed level of error decreases. One conclusion that we can draw, then, is that an interface with a large measure and inducing solutions that are sufficient regular should be selected. Unfortunately, the cost of computing the required number of interface modes may be prohibitive. The reader is referred to $[7,8]$ for error bounds that consider the discretization error. We remark that the eigenspace error depends upon the error due to modal truncation and discretization.

A variation on a fixed-interface CMS method is the automated multilevel substructuring (AMLS) method where the structure is recursively divided into thousands of substructures and associated interfaces. AMLS is motivated by the realization that CMS can be applied to the projections of (3) onto the subdomains $\Omega_{i}$ so resulting in a hierarchy $H^{1}(\Omega)$ orthogonal subspaces. The paper [3] examined a mathematical basis for AMLS in the continuous variational setting and the resulting algebraic formulation. Unfortunately, AMLS is not well suited to 3D eigenvalue problems when solid elements are used. The reason is that AMLS supposes that the interface matrices are formed and factored so that the cost of AMLS is that of computing a sparse direct factorization of the stiffness matrix using multifrontal methods. As is well known, sparse direct methods are not scalable with respect to mesh or the number of processors.

A alternative to AMLS is to not subdivide the interface into a hierarchy and instead consider one interface. Indeed, most modern graph partitioners generate a multitude of subdomains with one interface. A preconditioner for the Schur complement can be used within a preconditioned eigensolver for the interface eigenvalue problem. Although the interface problem is reduced in size over that of (3), the application of the mass and Schur complements matrices, and Schur complement 
preconditioners is expensive. Moreover, there remains the issue of maintaining the numerical orthogonality of the interface vectors and then, finally performing the Rayleigh-Ritz analysis. These observations are based on preliminary work by the authors when using the substructuring preconditioner [14] for the interface eigenvalue problem ${ }^{3}$.

There are other CMS techniques that are distinguished by how the interface is handled. MacNeal [34] introduced a Lagrange multiplier to enforce compatibility of the interface displacements that are referred to as free-free methods. For a one dimensional model problem, Bourquin [6] showed that a fixed interface method better approximates the eigenspace than a free-free method. The recent paper [33] by Rixen reviews several CMS techniques and introduces a dual fixed-interface method. The paper [10] considers replacing nonoverlapping CMS methods with overlapping methods. More work is needed in understanding the effect of the interface upon the approximation of the global modes, in particular approximations of the mass interface operator. The reader is referred to the thesis by Namar (under the supervision of Bourquin) that considers such an analysis. If efficient and accurate approximations of the interface operators were available, then an iteration between the substructures is possible. This leads us to discuss type two multilevel approaches.

The papers $[28,9]$ consider such approaches and provide references into the literature. Type two multilevel II approaches are motivated by the variational problem

$$
\min _{\mathbf{x}(\neq \mathbf{0})} \frac{\mathbf{x}^{T} \mathbf{K} \mathbf{x}}{\mathbf{x}^{T} \mathbf{M x}}
$$

on a sequence of grids or partition of $\Omega$ into subdomains. For example, a sequence of grids exists when generated as part of a multigrid strategy, and $\Omega$ is partitioned into a union of non-overlapping subdomains as part of a domain decomposition method. Because the projection operators associated with transferring information between grids or partitions can be selected $\mathbf{K}$ orthogonal, orthogonality of approximate eigenvectors is easily maintained. In addition, an iteration is naturally defined because accuracy (estimated via the norms of residuals) can be estimated at the fine grid. However, this iteration necessarily involves orthogonalizations (either via Gram-Schmidt or a Rayleigh-Ritz analysis) of fine grid vectors when more than one eigenpair is needed.

Practical experience with computing many modes using type two multilevel approaches is lacking. The authors are currently considering an algebraic multigrid scheme or RQAMG method. This builds upon the work of $[28,19]$. The benefit is that no geometry of the problem is needed.

For both types of multilevel II approaches, an important research question is the sensitivity of the partition (or grids) computed. All of the existing approaches make use of geometry to define a physical interface or set of geometric meshes. They are well-defined independent of geometric considerations. (For example, the proposed RQAMG is an extension of the RQMG technique due to ([28]).) What the effect is of using an algebraic interface or restriction of (3) needs further investigation. We remark that for fixed-interface CMS methods, this questions can be posed as a question of regularity of the interface for the differential equation. For instance, the bound (14) reflects regularity through $\alpha$.

\footnotetext{
${ }^{3}$ We graciously acknowledge the use of prototype codes developed by Clark Dohrmann of Sandia National Laboratories.
} 


\section{Conclusions}

We started with a formal introduction of the origin of the eigenvalue problem in structural dynamics. We then discussed the resulting partial eigenvalue problem and accuracy issues. Two multilevel approaches were then introduced. Multilevel approach I reviewed using a multilevel preconditioner within an iteration for the eigenpairs of (3). Our discussion of multilevel II approaches focused on CMS methods. A characteristic of these methods is that orthogonalization of fine grid vectors is avoided. This cost is replaced by that of solving an interface eigenvalue problem. Instead, type two multilevel II approaches are more evocative of domain decomposition methods for partial differential equations where iterations among substructures or grids is undertaken.

\section{Acknowledgments}

We acknowledge the ongoing work of and discussions with our colleagues in the structural dynamics and computational mathematics departments of Sandia National Laboratories, Jeff Bennighof, Andrew Knyazev and Olof Widlund.

\section{References}

1. P. Arbenz, U. Hetmaniuk, R. Lehoucq, and R. S. Tuminaro, A comparison of eigensolvers for large-scale $3 d$ modal analysis using amg-preconditioned iterative methods, International Journal for Numerical Methods in Engineering, In press (2005).

2. I. BABUŠKa AND J. Osborn, Eigenvalue problems, vol. II of Handbook of numerical analysis, Elsevier, 1991, pp. 641-788.

3. J. K. Bennighof And R. B. LeHOUCQ, An automated multilevel substructuring method for eigenspace computation in linear elastodynamics, SIAM J. Scientific Computing, 25 (2004), pp. 2084-2106.

4. L. Bergamaschi, G. Pini, and F. Sartoretto, Approximate inverse preconditioning in the parallel solution of sparse eigenproblems, Numerical Linear Algebra with Applications, 7 (2000), pp. 99-116.

5. M. Bhardwaj, D. Day, C. Farhat, M. Lesoinne, K. Pierson, And D. RIXEN, Application of the FETI method to ASCI problems: Scalability results on one-thousand processors and discussion of highly heterogeneous problems, International Journal on Numerical Methods in Engineering, 47 (2000), pp. $513-536$.

6. F. Bourquin, Analysis and comparison of several component mode synthesis methods on one-dimensional domains, Numerische Mathematik, 58 (1990), pp. 11-34.

7. F. Bourquin, Synthèse modale et analyse numérique des multistructures élastiques, $\mathrm{PhD}$ thesis, Université Paris VI, 1991.

8. F. Bourquin, Component mode synthesis and eigenvalues of second order operators: Discretization and algorithm, Mathematical Modeling and Numerical Analysis, 26 (1992), pp. 385-423. 
9. T. F. Chan And I. Sharapov, Subspace correction multi-level methods for elliptic eigenvalue problems, Numerical Linear Algebra with Applications, 9 (2002), pp. $1-20$.

10. I. Charpentier, F. D. Vuyst, and Y. Maday, Méthode de synthèse modale avec une décomposition de domaine par recouvrement, C. R. Acad. Sci. Paris, Série I, 322 (1996), pp. 881-888.

11. R. D. Cook, D. S. Malkus, M. E. Plesha, and R. J. Witt, Concepts and applications of Finite Element Analysis, John Wiley \& Sons, Inc, 2002.

12. R. R. Craig, JR. And M. C. C. Bampton, Coupling of substructures for dynamic analysis, AIAA Journal, 6 (1968), pp. 1313-1319.

13. E. R. DAVIDSON, The iterative calculation of a few of the lowest eigenvalues and corresponding eigenvectors of large real-symmetric matrices, J. Comput. Phys., 17 (1975), p. 87.

14. C. R. Dohrmann, A preconditioner for substructuring based on constrained energy minimization, SIAM J. Scientific Computing, 25 (2003), pp. 246-258.

15. T. ERICSSON AND A. RuHE, The spectral transformation Lanczos method for the numerical solution of large sparse generalized symmetric eigenvalue problems, Mathematics of Computation, 35 (1980), pp. 1251-1268.

16. C. Farhat, M. Lesoinne, and K. Pierson, A scalable dual-primal domain decomposition method, Numer. Lin. Alg. Appl., 7 (2000), pp. 687-714.

17. C. Farhat And F.-X. Roux, A Method of Finite Element Tearing and Interconnecting and its Parallel Solution Algorithm, Int. J. Numer. Meth. Engrg., 32 (1991), pp. 1205-1227.

18. Y. FENG AND D. OWEn, Conjugate gradient methods for solving the smallest eigenpair of large symmetric eigenvalue problems, International Journal on Numerical Methods in Engineering, 39 (1996), pp. 2209-2229.

19. T. FRIESE, Eine Mehrgitter-Methode zur Lösung des Eigenwertproblems der komplexen Helmholtzgleichung, PhD thesis, Freie Universität Berlin, 1998.

20. R. G. Grimes, J. G. Lewis, And H. D. Simon, A shifted block Lanczos algorithm for solving sparse symmetric generalized eigenproblems, SIAM J. Matrix Analysis and Applications, 15 (1994), pp. 228-272.

21. M. R. Hestenes And W. Karush, A method of gradients for the calculation of the characteristic roots and vectors of a real symmetric matrix, Journal of Research of the National Bureau of Standards, 47 (1951), pp. 45-61.

22. W. C. HuRTY, Vibrations of structural systems by component-mode synthesis, Journal of the Engineering Mechanics Division, ASCE, 86 (1960), pp. 51-69.

23. A. V. KNYAZEV, Preconditioned eigensolvers — an oxymoron, 7 (1998), pp. 104123.

24. — Toward the optimal preconditioned eigensolver: Locally optimal block preconditioned conjugate gradient method, SIAM J. Scientific Computing, 23 (2001), pp. $517-541$.

25. A. V. KNYAZEV AND K. NEYMEYR, Efficient solution of symmetric eigenvalue problems using multigrid preconditioners in the locally optimal block conjugate gradient method, 7 (2003), pp. 38-55.

26. R. B. Lehoucq, D. C. Sorensen, and C. Yang, ARPACK USERS GUIDE: Solution of Large Scale Eigenvalue Problems with Implicitly Restarted Arnoldi Methods, SIAM, Phildelphia, PA, 1998.

27. D. E. Longsine And S. F. MCCormik, Simultaneous Rayleigh-quotient minimization for $A x=\lambda B x$, Linear Algebra and Its Applications, 34 (1980), pp. 195-234. 
28. J. MAndel AND S. MCCormick, A multilevel variational method for $a u=\lambda b u$ on composite grids, Journal of Computational Physics, 80 (1989), pp. 442-452.

29. K. J. Maschhoff And D. C. Sorensen, P_ARPACK: An efficient portable large scale eigenvalue package for distributed memory parallel architectures, in Applied Parallel Computing in Industrial Problems and Optimization, J. Wasniewski, J. Dongarra, K. Madsen, and D. Olesen, eds., vol. 1184 of Lecture Notes in Computer Science, Berlin, 1996, Springer-Verlag.

30. Y. Notay, Combination of Jacobi-Davidson and conjugate gradients for the partial symmetric eigenproblem, Numer. Lin. Alg. Appl., 9 (2002), pp. 21-44.

31. E. Ovtchinnikov, Convergence estimates for the generalized Davidson method for symmetric eigenvalue problems $i$ : The preconditioning aspect, SIAM J. Numer. Anal., 41 (2003), pp. 258-271.

32. — Convergence estimates for the generalized Davidson method for symmetric eigenvalue problems $i$ : The subspace acceleration, SIAM J. Numer. Anal., 41 (2003), pp. 272-286.

33. D. J. Rixen, A dual craig-bampton method for dynamic substructuring, J. Comput. Appl. Math., 168 (2004), pp. 383-391.

34. R.MacNeal, A hybrid method of component mode synthesis, Computers and Structures, 4 (1971), pp. 581-601.

35. P. SESHU, Substructuring and component mode synthesis, Shock and Vibration, 4 (1997), pp. 199-210.

36. G. L. G. SleiJPen AND H. VAN DER Vorst, A Jacobi-Davidson iteration method for linear eigenvalue problems, SIAM J. Matrix Analysis and Applications, 17 (1996), pp. 401-425.

37. G. Strang And G. Fix, An Analysis of the Finite Element Method, PrenticeHall, englewood cliffs, nj ed., 1973. 